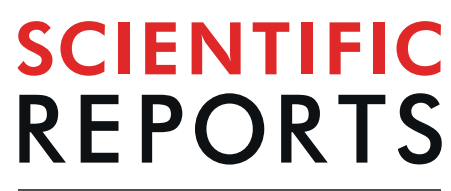

natureresearch

Check for updates

\title{
WFhb1-1 plays an important role in resistance against Fusarium head blight in wheat
}

\author{
Bimal Paudel ${ }^{1,7}$, Yongbin Zhuang ${ }^{1,2,7}$, Aravind Galla ${ }^{1,3}$, Subha Dahal ${ }^{1,4}$, Yinjie Qiu $\mathbb{B}^{1,5}$, \\ Anjun Ma $\mathbb{D}^{1,6}$, Tajbir Raihan ${ }^{1}$ \& Yang Yen $\mathbb{B}^{1 凶}$
}

Fusarium head blight (FHB) is a severe disease of wheat (Triticum aestivum L.). $Q$ fhb1 is the most important quantitative trait locus $(\mathrm{QTL})$ for $\mathrm{FHB}$ resistance. We previously identified wheat gene WFhb1-1 (aka WFhb1-c1) as a candidate for FHB resistance gene. Here we report that WFhb1-1 has been cloned. The gene (GenBank \# KU304333.1) consists of a single exon, encoding a putative membrane protein of 127 amino acids. WFhb1-1 protein produced in Pichia pastoris inhibits growth of both $F$. graminearum and $P$. pastoris in culture. Western Blotting with anti- WFhb1-1 antibody revealed a significant decrease $(p<0.01)$ in WFhb1-1 accumulation, 12 hours post Fusarium inoculation in nonQfhb1-carrier wheat but not in Ofhb1-carrier wheat. Overexpressing WFhb1-1 in non-Ofhb1-carrier wheat led to a significant decrease $(p<0.01)$ in Fusarium-damaged rachis rate, Fusarium-diseased kernel rate and DON content in harvested kernels, while silencing WFhb1-1 in Qfhb1-carrier wheat resulted in a significant increase $(p<0.01)$ in FHB severity. Therefore, WFhb1-1 is an important FHB resistance gene with a potential antifungal function and probably a key functional component of $Q$ fhb1 in wheat. A model regarding how WFhb1-1 functions in FHB resistance/susceptibility is hypothesized and discussed.

Fusarium head blight (FHB, also called scab or head scab), is a severe fungal disease of small grains such as bread wheat (Triticum aestivum L.), durum wheat (T. durum L.), oat (Avena sativa), and barley (Hordeum vulgare L.). FHB can be caused by several Fusarium species with F. graminearum as the primary pathogen in warm and humid regions worldwide including USA. Economic losses caused by FHB in wheat alone have been over billions of US dollar since $1990^{1,2}$. Reduced yields, shriveled grains, mycotoxin contamination, and reduction in seed quality are major factors that are related to the losses due to this disease $\mathrm{e}^{3-6}$. The mycotoxins produced by the pathogen remain in processed foods causing health hazards in humans and animals ${ }^{7}$. Deoxynivalenol (DON) is the primary mycotoxin produced by F. graminearum in infected grains ${ }^{3}$.

Utilization of host resistance to develop resistant cultivars is the most promising approach to control FHB. Two major types of FHB resistance are widely accepted: resistance to the initial infection (Type I), and resistance to the spread of infection in the spike (Type II) ${ }^{8}$. Type I resistance is common in barley but rare in wheat, which is most likely contributed by spike morphology $y^{9}$ and by activation of systemic innate immune responses ${ }^{10}$. In contrast, Type II resistance is attributed by different resistant genes, and has been more extensively studied and utilized. FHB resistance in wheat is a quantitative trait. Numerous genetic studies on various resistance sources have shown that Type II resistance in each resistant wheat cultivar is most likely controlled by two to three major genes and a few minor genes ${ }^{11,12}$. Molecular mapping of quantitative trait loci (QTLs) for Type II resistance has been extensively reported. Overall, about 100 QTLs associated with FHB resistance are mapped in all wheat chromosomes but 7D ${ }^{13}$. Effectiveness of these QTLs is strongly influenced by genetic background and environments. Efforts to identify candidate genes of some key QTLs have also been made ${ }^{14-21}$, which has led to a better understanding of the pathogenesis and the resistance mechanisms.

\footnotetext{
${ }^{1}$ Department of Biology and Microbiology, South Dakota State University, Brookings, SD, 57007, USA. ${ }^{2}$ Present address: College of Agronomy, Shandong Agricultural University, Taian, Shandong, 271018, China. ${ }^{3}$ Present address: Department of Entomology, University of Arkansas, Fayetteville, AR, 72701, USA. ${ }^{4}$ Present address: Department of Molecular Genetics, University of Toronto, Toronto, ON, M5S 1A8, Canada. ${ }^{5}$ Present address: Department of Horticultural Science, University of Minnesota, St. Paul, MN, 55108, USA. ${ }^{6}$ Present address: Department of Biomedical Informatics, College of Medicine, The Ohio State University, Columbus, OH, 43210, USA. ${ }^{7}$ These authors contributed equally: Bimal Paudel and Yongbin Zhuang. ${ }^{\varpi}$ e-mail: yang.yen@sdstate.edu
} 
The FHB-resistance QTL Qfhb1 (formerly known as Qfhs.ndsu-3BS and sometimes simply called Fhb1) on chromosome arm 3BS was first identified from Chinese cultivar Sumai $3^{22}$. Since then, it has been well defined as the most effective and the most stable QTL across different genetic backgrounds and various environment ${ }^{22-26}$. $Q f h b 1$ usually account for $20 \sim 60 \%$ of the phenotypic variation in FHB resistance ${ }^{13}$. Therefore, Qfhb1 has been the main resistance QTL deployed in wheat breeding to improve FHB resistance worldwide and the research focus for the resistance mechanism in wheat.

Fine mapping efforts have indicated that $Q f h b 1$ contains a complex chromosomal region dissimilar in the sequence between wheat accessions (see the review by Paudel and $\mathrm{Yen}^{27}$ ). In wheat cultivar Chinese Spring, this QTL was narrowed down to a $261-\mathrm{kb}$ region of wheat chromosome arm $3 \mathrm{BS}^{14,28-30}$. Seven potential genes in this QTL region have been recognized, cloned and evaluated, but none of them was found to be an FHB resistance gene $^{14}$. Later, 28 genes were recognized in a Sumai 3-derived, Qfhb1-containing 860-kb region, from which only a GDSL lipase gene showed a pathogen-dependent expression pattern and thus was thought to be qualified as a functional gene candidate for $Q f h b 1$ while a possibility of more than one gene causing the phenotypic difference was also suggested ${ }^{16}$. However, this GDSL was not among the 13 genes identified in the QTL interval of Sumai 3 by Pumphrey ${ }^{31}$ and Rawat et al. ${ }^{17}$. Rawat et al. ${ }^{17}$ instead claimed that a pore forming toxin like protein gene (PFT) is a functional gene of $Q f h b 1$. Nevertheless, $Q f h b 1$ is able to detoxify $D O N^{32,33}$, but $P F T$ cannot $^{17}$. The unique existence of GDSL and PFT in Qfhb1-carrier wheat genotypes was a key reason for their identification as the genic component of $Q f h b 1$. Su et al. ${ }^{19}$ and Li et al.$^{20}$ independently did an extensive study by surveying hundreds of wheat accessions from worldwide collections and found that neither PFT nor GDSL is unique to Qfhb1-carrier wheat genotypes. Additionally, He et al. ${ }^{34}$ and Jia et al. ${ }^{35}$ also reported that PFT exists and functions in some susceptible wheat accessions they surveyed. These latest studies called into question the idea of PFT being an Fhb1 candidate gene. In the most recent publications, Su et al. ${ }^{19}$ and Li et al ${ }^{20}$ independently concluded that a mutation of a histidine-rich calcium-binding protein gene [named as TaHRC in Su et al. ${ }^{19}$ and His in Li et al. ${ }^{20}$ ] confers resistance against FHB. TaHRC is a susceptible gene, and a large deletion in the start codon region of its susceptible allele makes it silent, resulting in FHB resistance ${ }^{19,36,37}$. However, Li et al ${ }^{20}$ claimed that the deletion in TaHRC had caused frameshift leading to expression of a new protein that confers resistance. Both Su et al. ${ }^{19}$ and Li et al. ${ }^{20}$ reported that the FHB resistance conferred by the deletions is genetically semi-dominant. Previously, we reported that a wheat gene with an unknown function, then temporarily named as Wheat Fhb1 candidate 1 (WFhb1-c1, WFhb1-1 or Fhb1-1), could be a functional genic component of this QTL ${ }^{15,23,38}$. Therefore, the debate on $Q f h b 1$ 's genic component is going on.

Various functional mechanisms of $Q f h b 1$ have also been proposed, but none has been validated without argument. These proposed functions include detoxifying $\mathrm{DON}^{32,33}$, thickening secondary cell wall in rachises after pathogen infection to prevent the pathogen to spread ${ }^{39}$, inhibiting pectin methyl esterase to prevent the pathogen from penetrating the host cell wall ${ }^{15}$, mediating jasmonic acid (JA) and ethylene (ET) signaling pathways to elicit local and systemic resistance ${ }^{16,38,40,41}$, killing the infecting pathogen ${ }^{17,42}$ or simply reducing FHB susceptibility that leads to FHB development ${ }^{37,43}$. Possible simultaneous regulation of at least two different resistance mechanisms by multiple functional components of $Q f h b 1$ has also been suggested ${ }^{16,29,40,43}$. Nevertheless, $Q f h b 1$ has been well recognized to simultaneously reduce FHB severity in the spikes and DON content in the kernels.

As mentioned above, our previous studies using a combination of approaches including transcriptomics and physiological studies ${ }^{38}$, and QTL, eQTL, and physical mappings ${ }^{15,23}$ have revealed wheat gene WFhb1-1 as a candidate for the functional genic component of $Q f h b 1$ in Sumai 3. Analyzing the expression pattern of this gene revealed its differential expression between FHB-resistant and FHB-susceptible wheat lines in response to the F. graminearum infection during the early stage of the pathogenesis ${ }^{15}$. This result implies that the pathogen can suppress the expression of this wheat gene to initiate FHB pathogenesis in FHB-susceptible wheat genotypes, but such suppression mechanism does not work in FHB-resistant wheat genotypes. Therefore, cloning of this gene, and elucidating its function are necessary to further our understanding about its role in FHB pathogenesis/resistance and about how it is regulated by the pathogen infection. Here we report the results of our efforts to clone this gene from Sumai 3, to elucidate its biological function and to functionally validate its role in FHB resistance using Sumai 3 and a pair of Sumai 3-derived Qfhb1 near-isogenic lines 206-1-1-2 (carrying Qfhb1, called NIL-R hereafter) and 260-1-1-4 (not carrying Qfhb1, called NIL-S hereafter).

\section{Results}

Cloning the full-length cDNA and the genomic sequence of WFhb1-1. The first step of this study was to clone the full-length cDNA of WFhb1-1, the candidate gene for a functional genic component of $Q f h b 1$ which we identified in our previous study ${ }^{15}$. The $5^{\prime}$ and the $3^{\prime}$ ends of the cDNA were first cloned from the spike sample of Sumai 3 with $5^{\prime} / 3^{\prime}$ RACE technologies, respectively, and the full-length cDNA sequence was then formed by merging the two partial cDNA fragments together. The full-length cDNA was then confirmed by cloning the full sequence from total RNA of Sumai 3 spikes by RT-qPCR and sequencing. The full-length cDNA sequence (Fig. 1A) has been deposited in GenBank (GenBank \# KU304333.1).

The corresponding genomic sequence was then cloned from Sumai 3 by PCR with gene-specific primers designed on the basis of the cDNA sequences and the corresponding up-stream and down-stream genomic sequences of chromosome arm 3BS pseudomolecule (GenBank \#: HG670306.1) of wheat cultivar Chinese Spring (now part of wheat reference genomic sequence IWGSC RefSeq v1.0.). Comparing the full-length cDNA and the genomic coding sequence revealed only one exon in this gene (Fig. 1A). Analyzing the upstream genomic sequence revealed two potential promoters (Fig. 1A). Our in silico mapping has located the cloned gene between the $5641624 \mathrm{bp}$ and the $5642007 \mathrm{bp}$ in the 3BS pseudomolecule flanked by markers Xsts9-2 and Xsts65-3 (Fig. 1B).

The predicted protein of this cloned gene (GenBank \# ANE31719.1) has 127 amino acid residues, which shows $98 \%$ identity $(E=1 \mathrm{e}-80)$ to an unnamed wheat protein (GenBank \#: CDM801516.1) reported by Choulet et al. ${ }^{44}$. Analyzing the predicted protein with Phobius (http://phobius.sbc.su.se) predicted that it is probably a 


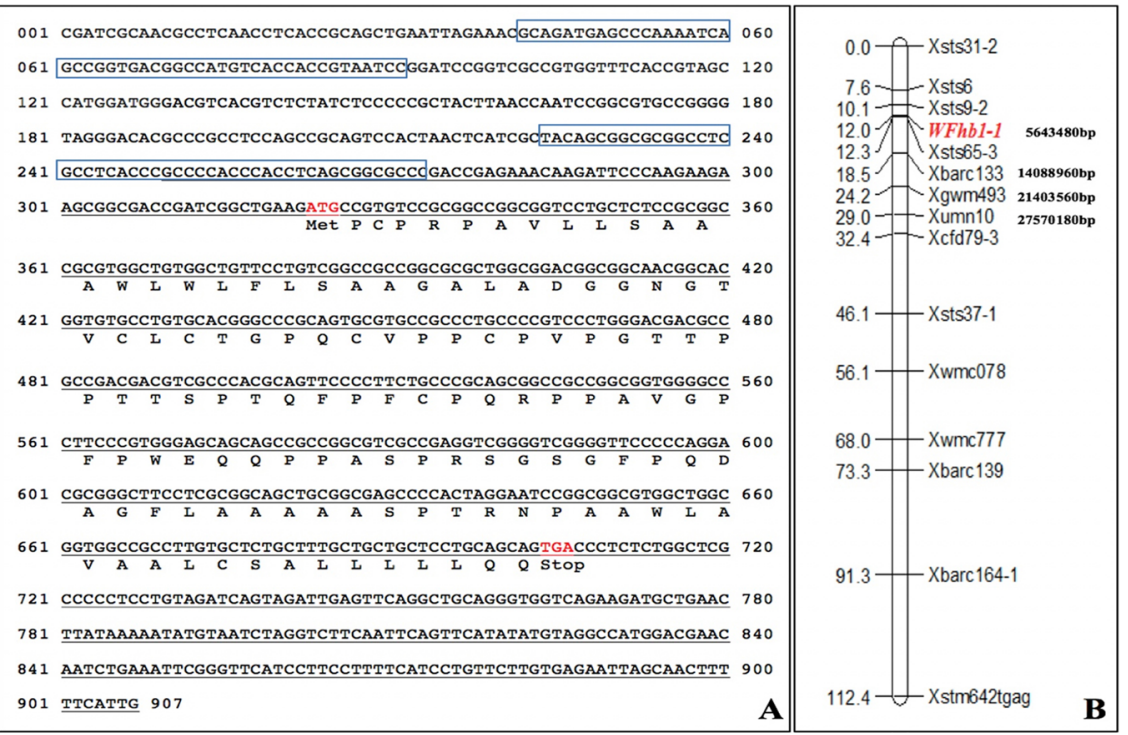

Figure 1. Sequences and map position of WFhb1-1. (A) Genomic sequence of WFhb1-1 with the two predicted promoter regions in blue squares. The full-length cDNA is underlined and the predicted protein sequence is underneath. (B) In silico mapping of WFhb1-1 to Chinese Spring chromosome 3B pseudomolecule with position in the sequence indicated.

transmembrane protein with an undefined extracellular signaling domain (Supplementary Fig. S1). However, analyzing it with ScanProsite (https://prosite.expasy.org/scanprosite/) did not give a clear clue on its biological function since no obvious conservative protein domain was found in the predicted protein. Therefore, we need to reveal the biological function of this protein experimentally.

Protein expression in Pichia pastoris. To study the biological function of the cloned gene, its open reading frame (ORF) was cloned into and expressed in P. pastoris (Supplementary Fig. S2). Two antibodies PA-1 and PA-2 were designed and produced for specific detection of the putative protein encoded by the cloned gene (Fig. 1A). The yeast-produced WFhb1-1 was detected in the total protein extracted from the expressing yeast strain X33:T by Western blotting using PA-1 as the primary antibody (Fig. 2A). The yeast-produced WFhb1-1 stays intracellularly as it was detected in pellet samples but not in the supernatant of the cell extraction (Fig. 2D). The mass of the protein was estimated to be $\sim 60 \mathrm{kDa}$ (Fig. 2A-D), which is about 4 times larger than the $\sim 13 \mathrm{kDa}$ estimated from the predicted amino acid sequence. This observation indicate that this wheat protein probably forms a tetramer in the yeast total protein extraction. Alternatively, the yeast-produced WFhb1-1 may tightly bind to another yeast protein in the yeast cells.

We have noticed that the yeast production of WFhb1-1 could be detected only up to Day 2 of the induced expression (Fig. 2D), and growth of X33:T (indicated by its increased cell number per cubic unit of the culture) also occurred only during the first two days, while the control strain X33:00 continued to grow (Fig. 2E). These results suggest that the yeast-produced wheat protein may be toxic to P. pastoris itself so that it stops growing and ceases the production when WFhb1-1 concentration reaches the life-threatening threshold in two days.

In vitro inhibition assay of $F$. graminearum growth with the yeast-produced WFhb1-1. Since the yeast-produced WFhb1-1 showed a potential anti-yeast activity in the expression system (Fig. 2E), we conducted experiments to see if it could also inhibit F. graminearum from growing in culture. Our data indicate that growth of F. graminearum in potato dextrose broth was indeed inhibited when $500 \mu \mathrm{g} / \mathrm{mL}$ or more of the total protein extracted from X33:T was added into the culture (Fig. 2F, G, Supplementary Fig. S3). The observed growth inhibition of F. graminearum by the total protein extracted from X33:00 yeast strain compared to the sterile water could be caused by precipitation reagent residues in the total protein sample. Nevertheless, the obvious difference in inhibiting F. graminearum growth by the X33:T total protein compared to that by the X33:00 total protein indicates that the yeast-produced WFhb1-1 seems to have a broad antifungal ability, which could reduce FHB development in wheat. Further research in this aspect with purified WFhb1-1 is needed to further explore its anti-fungal mechanism and its potential utility as a bio-fungicide for controlling fungal diseases.

Evaluation of WFhb1-1 protein accumulation in wheat spikes. Western blotting assays using either anti-WFhb1-1 antibodies PA-1 (Fig. 2C) or PA-2 (Fig. 3) as primary antibody have detected WFhb1-1 accumulation in wheat spikes, confirming WFhb1-1 as a protein coding wheat gene. Our previous study showed that WFhb1-1 is suppressed by the pathogen infection in FHB-susceptible wheat genotypes but not in the FHB-resistant genotypes ${ }^{15}$. We would like to know how this transcription suppression impacts WFhb1-1 protein accumulation in vivo. Protein samples were collected from the F. graminearum-inoculated and the mock-inoculated spikelets of the NIL pair at 0,8,12, 24, 36 and 48 hpfi (hours post Fusarium infection) and subjected to Western blotting using anti-WFhb1-1 antibody PA-2 as the primary antibody (Fig. 3; Supplementary 


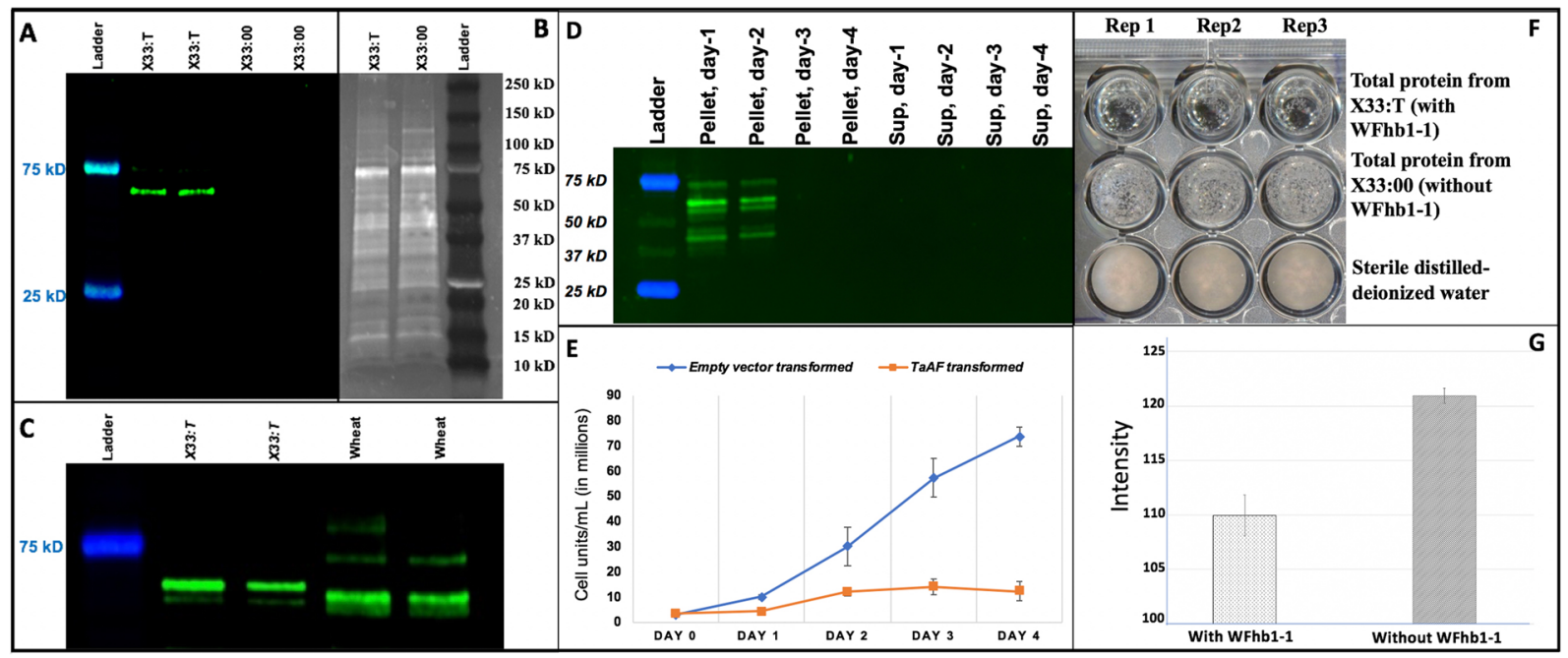

Figure 2. Photos and graphics showing the results of elucidating the WFhb1-1 produced by WFhb1-1expression Pichia pastoris strain X33:T. (A) a photo of a Western Blot of total protein isolated from X33:T and the wild type $P$. pastoris X33:00 probed with anti- WFhb1-1 antibody PA-2; (B) a photo of a Sypro-Ruby stain-polyacrylamide gel of the total protein isolated from X33:T and X33:00; (C) a photo of a Western Blot of total protein isolated from X33:T and wheat spikes probed by PA-1. (D) A photo of Western Blot of total protein extracted from X33:T probed with anti- WFhb1-1 antibody PA-1 on day 1, day 2, day 3 and day 4 after methanol was added into the culture to turn on WFhb1-1 expression; Sup: supernatant. (E) A graphic showing the growth kinetics of X33:T and X33:00 post addition of methanol into the culture medium to turn on WFhb1-1 expression. (F) A photo showing growth of Fusarium graminearum in $100 \mu \mathrm{L}$ potato dextrose broth supplemented with $500 \mu \mathrm{g} / \mathrm{mL}$ total protein isolated from X33:T or X33:00 or sterile water. About 1000 conidia were used to initiate the culture in each well. The photo was taken two weeks after the culture started. (G) Comparison of intensity of fungal growth between WFhb1-1 protein-added and control protein-added wells.
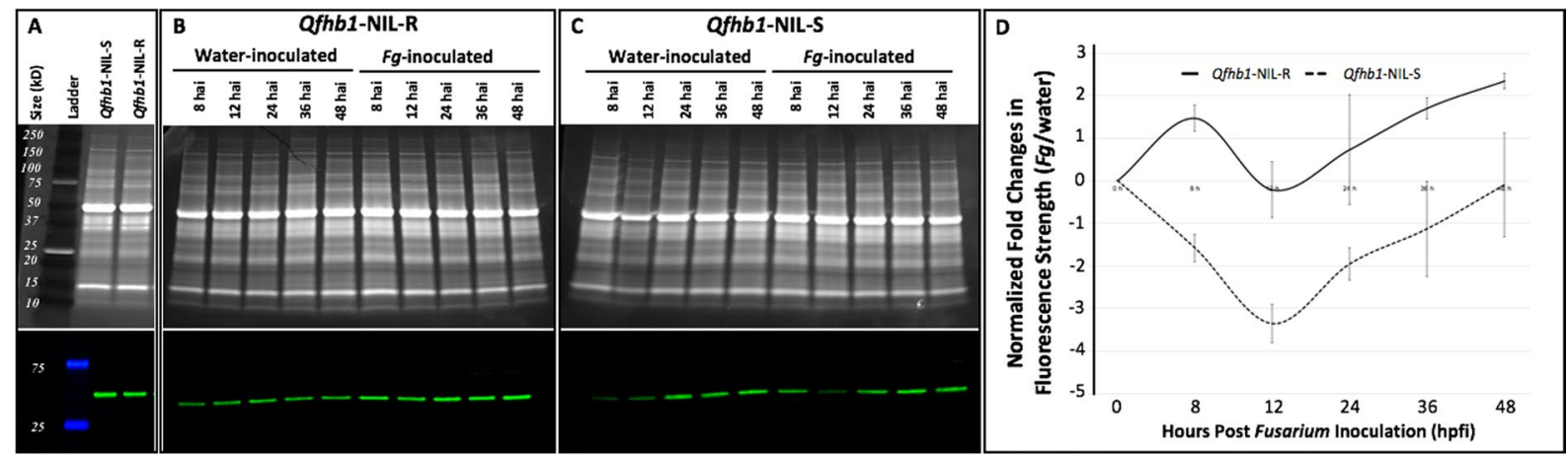

Figure 3. Photos and graphic showing the results of Western Blotting of total proteins isolated from wheat spikelets inoculated with Fusarium graminearum $(\mathrm{Fg})$ or water at $0,8,12,24,36$ and 48 hpfi (hours post Fusarium inoculation). In A, B and C, the upper panels are images of polyacrylamide gel stained with SYPRO Ruby, and the lower panels are photos of Western Blot probed with probed with anti- WFhb1-1 antibody PA-2. (A) Total protein isolated from the water-inoculated spikelets of Qfhb1-NIL-R and Qfhb1-NIL-S at 0 hpfi; (B) total protein isolated from the $F g$ - and the water-inoculated spikelets of $Q f h b 1-N I L-R ;(C)$ total protein isolated from the Fg-and the water-inoculated spikelets of Qfhb1-NIL-S; (D) Normalized fold changes in fluorescence strength of WFhb1-1 revealed by Western blotting between the Fg-inoculated and the water-inoculated spikelets of each NIL at the six time points. The normalization was done by setting the value at 0 hpfi as the baseline for comparisons.

Figs. S4 and S5, Supplementary Table S1). We did not observe a significant difference at any time point between the FHB-inoculated and the mock inoculated samples of NIL-R (Fig. 3B,D, Supplementary Table S1). However, significant difference $(p<0.01)$ was observed at $12 \mathrm{hpfi}$ between the FHB-inoculated and the mock-inoculated NIL-S spikes (Fig. 3C,D). These observations suggest that the pathogen infection reduces WFhb1-1 accumulation at the early stage of FHB pathogenesis but only in the FHB-susceptible wheat, similarly as we previously observed at the transcription level ${ }^{15}$. The estimated mass of the in vivo-produced WFhb1-1 is $\sim 45 \mathrm{kDa}$ (Figs. 2C \& $3 \mathrm{~A}$ ), which is about three times larger than the $\sim 13 \mathrm{kDa}$ calculated from WFhb1-1 polypeptide sequence but smaller than the $\sim 60 \mathrm{kDa}$ estimated for the yeast-produced WFhb1-1 (Fig. 2C). The cause for the size difference between 


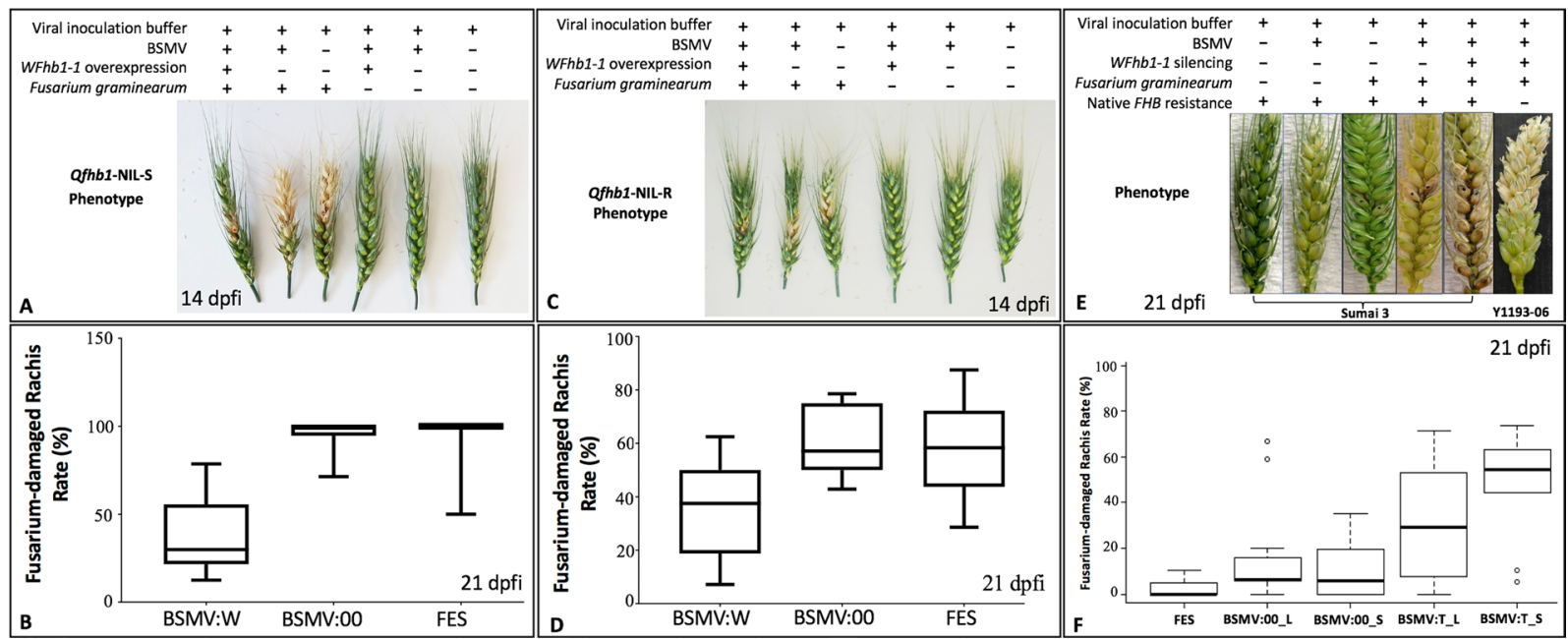

Figure 4. Photos and graphics showing the results of overexpression of WFhb1-1 in near-isogenic lines Qfhb1-NIL-S (A,B) and Qfhb1-NIL-R (C,D) or silencing of WFhb1-1 in FHB-resistant cultivar Sumai 3 (E,F) and FHB-susceptible landrace Y1193-06 (E) using a barley-stripe-mosaic virus (BSMV)-based system. Viral inoculation on spikes was applied. dpfi: days post Fusarium inoculation; FES: viral inoculation buffer FES; BSMV:00: wildtype BSMV; BSMV:W: WFhb1-1-overexpressing BSMV; BSMV:T: WFhb1-1-silencing BSMV; _L leaf inoculation of BSMV; _S: spike-inoculation of BSMV. The red or black dots on spikelets indicate the inoculated spikelets.

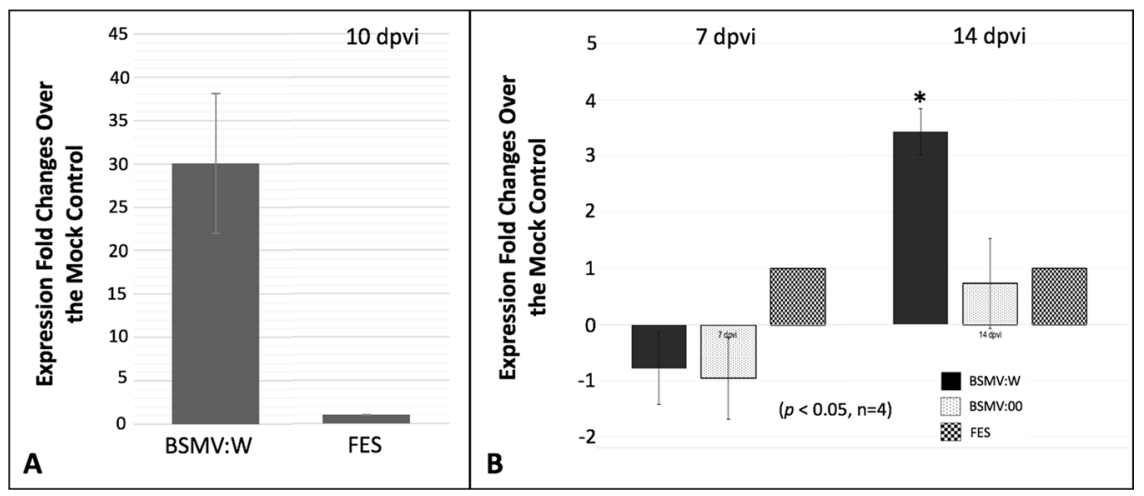

Figure 5. Results of RT-qPCR assays of WFhb1-1 transcript abundance changes in leaves (A) or spikes (B) of the Qfhb1-NIL-S plants inoculated with the WFhb1-1-overexpressing strain (BSMV:W), wildtype BSMV (BSMV:00) in comparison with the viral inoculation buffer FES-inoculated mock control; dpvi: days post viral inoculation. Four biological repeats per treatment and three technical repeats per biological repeat were included in the analysis.

the yeast-produced, the wheat-produced and the calculated WFhb1-1 is unknown but could be due to formation of different multimers (i.e. trimer in wheat vs. tetramer in yeast). Binding to a protein of different size in the yeast vs. in wheat is an alternative explanation.

WFhb1-1 gene overexpression in wheat. To understand the function of WFhb1-1 in FHB resistance, we applied a barley stripe mosaic virus (BSMV)-based, virally-induced gene overexpression system to NIL-S and NIL-R to see how overexpression of WFhb1-1 will impact FHB susceptibility/resistance in the NILs. To optimize this system in the NILs, the $3^{\text {rd }}$ leaves of 10-day old wheat seedlings were inoculated with either WFhb11 -overexpressing BSMV strain BSMV:W or FES viral inoculation buffer. We first examined the existence of BSMV:W and the WFhb1-1 expression by RT-qPCR in newly emerged leaves of the inoculated plants at $10 \mathrm{dpvi}$ (days post viral inoculation). The BSMV was successfully detected in the BSMV:W-inoculated wheat plants but not in the FES-inoculated plants by RT-PCR, indicating that the infected BSMV:W was successfully assembled within the infected wheat leaves and were able to move to other parts of the plants. Since BSMV is known to replicate in the chloroplasts of the host cells, some degree of chlorosis can be seen in the BSMV-infected plant tissue (Fig. 4E) if the virus number is high. However, such chlorosis has minimum impact on the plant tissues and has never caused tissue necrosis under the experimental conditions.

We detected up to 30 fold increase WFhb1-1 expression in BSMV:W-inoculated NIL-S plants compared to the FEF-inoculated NIL-S plants (Fig. 5A). This observation confirms that BSMV:W infection can induce 


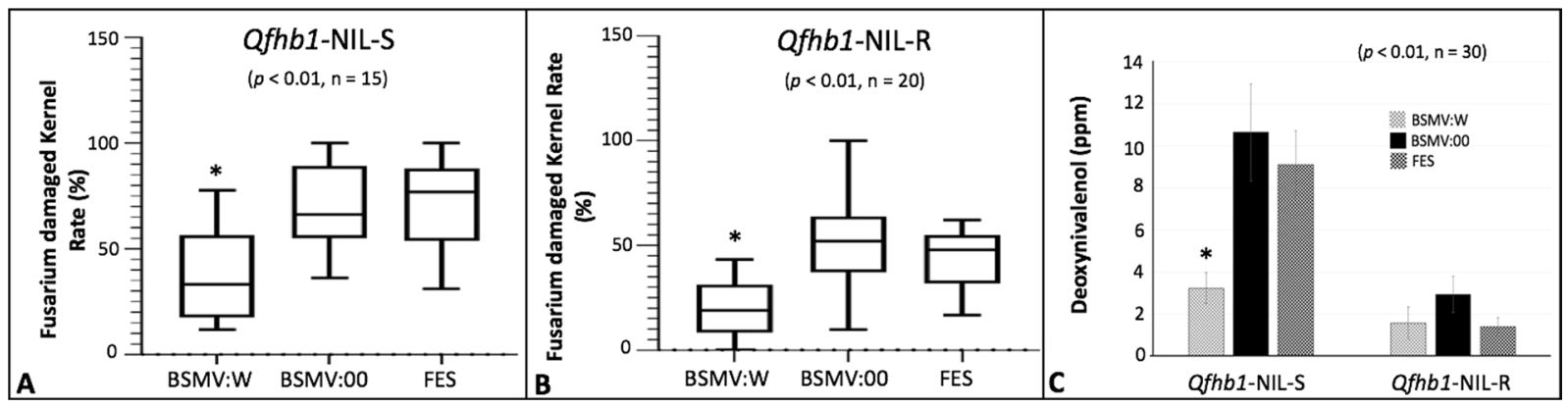

Figure 6. Graphics showing mean Fusarium-damaged kernel rate (\%) (A,B) and deoxynivalenol content (ppm) (C) in the harvested kernels of Qfhb1-NIL-S and Qfhb1-NIL-R inoculated either with WFhb1-1-overexpressing BSMV:W, the wildtype BSMV:00 or the viral inoculation buffer FES. *Significantly different compared with others.

overexpression of WFhb1-1 in the infected NIL-S plants. We also tested direct BSMV:W inoculation in spikes of NIL-S plants when three fourth of a spike emerges from the flag leaf. In this experiment, wheat plants inoculated with BSMV:00 (the wildtype BSMV strain) or FES were used as the controls. We observed significant induction of the WFhb1-1 overexpression in the spikes of the BSMV:W-inoculated plants compared to the BSMV:00- or the FES-inoculated plants (Fig. 5B). Results of this spike inoculation experiment suggest that significant overexpression could be detected at 14 dpvi.

To understand if WFhb1-1 indeed plays a role in FHB resistance in wheat, we visually observed FHB severity (Fig. 4A,C) on the inoculated spikes, and investigated Fusarium damaged rachides (FDR) rate (Fig. 4B,D, Supplementary Fig. S6), Fusarium damaged kernels (FDK) rate (Fig. 6A,B) and DON content in harvested kernels (Fig. 6C, and Supplementary Table S2) in the WFhb1-1-overexpressing wheat spikes under a high disease pressure (two spikelets were inoculated instead of usually one). For this purpose, NIL-S and NIL-R plants that were pre-inoculated with BSMV:W, BSMV:00 or FES were inoculated with F. graminearum conidia or water, respectively.

FDR data was collected from all the treated plants at 7, 14, 21, and $28 \mathrm{dpfi}$ and analyzed (Supplementary Fig. S5). One-way ANOVA and T-test both showed that the FDR was significantly lower $(p<0.01)$ in the BSMV:W-inoculated spikes compared to the BSMV:00- or the FES-inoculated spikes in all the four time points for NIL-S and three of the four time points for NIL-R; FDR at 7 dpfi for NIL-R was not statistically significant (Supplementary Fig. S5). Similarly, FDK data was also collected for all the treated plants and it was also found significantly lower $(p<0.01)$ in the BSMV:W-inoculated spikes than in the BSMV:00- or the FES-inoculated spikes for both NILs (Fig. 6A,B). For NIL-S, the DON level was found significantly lower in the BSMV:W-inoculated spikes compared to the BSMV:00- or the FES-inoculated spikes, while no difference was observed among the treatments of NIL-R (Fig. 6C, Supplementary Table S2). It seems that overexpressing WFhb1-1 in NIL-S reduces FHB to a level comparable to that observed in NIL-R (Figs. 5A,B \& 6; Supplementary Fig. S6 and Table 2).

WFhb1-1 gene silencing in wheat. To further confirm WFhb1-1's role in FHB resistance in wheat, the BSMV-system was also applied to knock WFhb1-1 down in spikes of Sumai 3 and Y1193-06 with RNA interference. The reason for using these two wheat genotypes instead of the NILs in this experiment was that Sumai 3 has the strongest FHB resistance of all wheat genotypes that have been studied so far. It has not only Qfhb1 but also other major FHB-resistance QTL, such as Qfhb_6BL $L^{23}$, Qhfs.ifa-5A (aka. Fhb5) ${ }^{45-47}$ and Qfhb.mgb-2A ${ }^{21}$, and it has been the most used FHB resistance source in wheat improvement worldwide. WFhb1-1 could be a major FHB-resistance gene if knocking it down can significantly compromise FHB resistance in Sumai 3. Y1193-06 has the worst FHB susceptibility of all wheat genotypes we have evaluated so far, and, thus, is the best control in contrast to Sumai 3.

As we did in the WFhb1-1 overexpression assay, we first tested if the knockdown works in Sumai 3. The leaves of Sumai 3 were inoculated with BSMV:T (the WFhb1-1-silencing BSMV strain), BSMV:00 or FES, and WFhb1-1 expression was monitored at 7, 15 and 21 dpvi (Fig. 7A). Using leaves instead of spikelets here is totally for the convenience since we had already known that WFhb1-1 is expressed in the whole plant and that the BSMV vectors will systematically spread to the entire plant body after inoculation.

Significant down expression of WFhb1-1 was observed at 15 dpvi in the BSMV:T-inoculated plants compared to those inoculated with BSMV:00 or FES. WFhb1-1 mRNA abundance in the BSMV:T-inoculated plants was 59 97\% less than in the BSMV:00-inoculated plants. WFhb1-1 mRNA was 17.1 37.5\% less abundant in the BSMV:00-inoculated plants than in the FES-inoculated plants, which is statistically not significant $(p>0.05)$. As shown in Fig. 7B, the WFhb1-1-silencing insert in BSMV:T seems to be partially deleted from its viral carrier probably during the viral replication. This could explain why we observed a down-fall in WFhb1-1 silencing at 21 dpvi (Fig. 7A). Code optimization seems to be needed to make the insert stable.

Then FHB inoculation was applied to the BSMV:T-inoculated and the control plants to test if silencing WFhb11 in wheat spikes can increase FHB severity. In this experiment, BSMV:T was used for both leaf (this treatment was designated as BSMV:T_L) and spike (this treatment was designated as BSMV:T_S) inoculations in both Sumai 3 and Y1193-06. BSMV:00 and FES inoculations were conducted as the viral inoculation controls and sterile water was used as the mock Fusarium inoculant. FDR was monitored at 7, 15, 21 and 28 dpfi. Our data show 

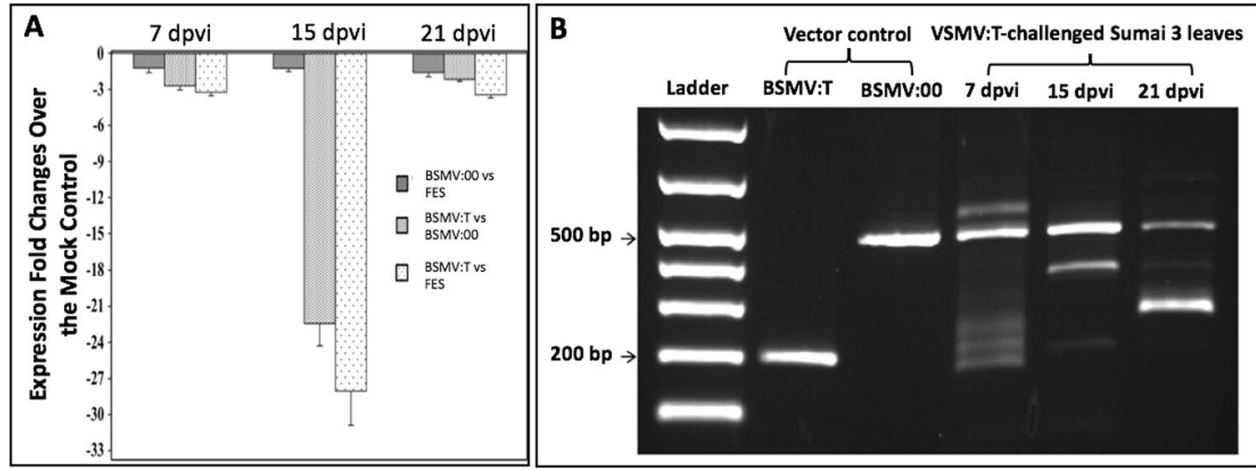

Figure 7. Results of RT-qPCR of WFhb1-1 silencing (A) or PCR (B) assays of the stability of WFhb1-1-insert in BSMV:T viral vector in leaves of inoculated Sumai 3. Wildtype BSMV:00 and FES were used as the controls; dpvi: days post viral inoculation.

that FHB resistance is compromised in the BSMV:T-treated Sumai 3 plants (Fig. 5E,F, Supplementary Figs. S6 to S8), which enabled F. graminearum to quickly spread to adjacent spikelets, whereas, FHB symptoms in the FESand the BSMV:00-treated Sumai 3 plants were mainly limited to the inoculated spikelets (Fig. 5E; Supplementary Fig. S8). This successful breakdown of Type II resistance by Fusarium infection in BSMV:T-treated Sumai 3 plants could be phenotypically observed as early as $7 \mathrm{dpfi}$ (Supplementary Fig. S8). In these plants, the disease seemed to spread mainly toward the base of the spikes, with only one or two upper rachis internodes being infected. Even at $28 \mathrm{dpfi}$, the upper spikelets remained clear of the fungal infection (Supplementary Fig. S8). As expected, no significant difference between treatments and control was observed in Y1193-06 (Fig. 6E; Supplementary Figs. S9 \& S10). We noticed that the spikelets above the inoculation site on the BSMV:T + Fusarium inoculated spikes of Y1193-06 were dry to death (Fig. 5E) while this phenomenon was not observed on the BSMV:00+FHB inoculated Y1193-06 spikes. Therefore, knocking WFhb1-1 down has enhanced FHB susceptibility in Y1193-06. Pearson's product-moment correlation test using mean value of WFhb1-1 abundance at $24 \mathrm{hpfi}$ and FDR index data at $7 \mathrm{dpfi}, 15 \mathrm{dpfi}$ and $21 \mathrm{dpfi}$ collected from Sumai 3 indicated a strong negative association between the two $(p=0.00027$ and Cor $=-8.62)$. Our data indicate that viral inoculation on spike apparently is more effective in inducing RNAi than the leaf-inoculation (Supplementary Figs. S7 \& S8).

Evaluation of WFhb1-1 expression in TaHRC-knockout Bobwhite mutant. CRISPR/Cas9-based gene editing of TaHRC in FHB-susceptible Bobwhite resulted in deletion mutations that knocked TaHRC out, gaining FHB resistance ${ }^{28}$. Expression of WFhb1-1, PFT, GDSL and TaHRC were compared between the TaHRC-knockout mutant created by Sue et al. ${ }^{19}$ and the wildtype of Bobwhite. Compared to the wildtype Bobwhite, TaHRC expression was found $\sim 2.5$ or $\sim 3.58$ folds lower, respectively using the gene-specific primers reported by Su et al. ${ }^{19}$ or Li et al..$^{20}$, in the spikelets of the knockout plants 24 hours after FHB inoculation, while the expression of WFhb1-1 was 3 folds higher in the knockout mutant plants. Whereas, in both Bobwhite lines, expression of $P F T$ was undetectable and GDSL expression level was too low to make any meaningful analysis. Therefore, both PFT and GDSL do not seem to have any role in the FHB resistance conferred by Qfhb1.

\section{Discussion}

The goal of this study is to identify the functional component of the major FHB resistance QTL Qfhb1 in wheat. We have taken a functional approach to reach this goal. We first conducted transcriptomics analysis between FHB-resistant and FHB-susceptible wheat lines and identified 637 genes that have significantly changed their expression abundance in wheat spikelets after F. graminearum infection and, therefore, are FHB-related genes ${ }^{38}$. Of these 637 genes, 406 genes which are associated with FHB resistance were analyzed by expression QTL (eQTL) mapping, and the significantly changed expression of three genes were found to be associated with $Q f h b 1^{15}$. Since these three genes can either physically locate in $Q f h b 1$ or physically locate at other places in the genome but are regulated by a gene in $Q f h b 1$, we conducted a physical mapping using a series of nullisomic-tetrasomic lines of Chinese Spring to identify their physical locations in wheat genome and found that WFhb1-1 is the only gene that is both physically located to wheat chromosome $3 \mathrm{~B}$ where $\mathrm{Q} f \mathrm{hbl}$ locates and functionally associated with $Q f h b 1^{15}$. In the present study, we have revealed the coding sequence of WFhb1-1 (Fig. 1), elucidated its expression at protein level (Figs. 2 and 3), and transiently assayed its function in FHB resistance with overexpression and RNAi-induced silencing (Figs. 4 and 5).

Data obtained in the present study shows that F. graminearum infection causes a significant reduction of WFhb1-1 accumulation in NIL-S in the early hours of the disease development but not in NIL-R (Fig. 3). This observation is in line with our previous observation at the transcription level showing negative regulation of WFhb1-1 by the infecting pathogen. Our data have also shown that overexpressing WFhb1-1 leads to a significant reduction in FHB severity under a high disease pressure in both the NILs, and the FHB resistance level in the WFhb1-1-overexpressing NIL-S plants is comparable to the level usually observed in NIL-R (Figs. 4A-D and 6A,B; Supplementary Fig. S6). By contrast, silencing WFhb1-1 leads to the significant compromise of FHB resistance in Sumai 3 and a noticeably increased FHB susceptibility in Y1193-06 (Fig. 4E,F; Supplementary Figs. S7 to S11). Overexpressing WFhb1-1 also significantly reduces DON content in the kernels of NIL-S (Fig. 6C). 


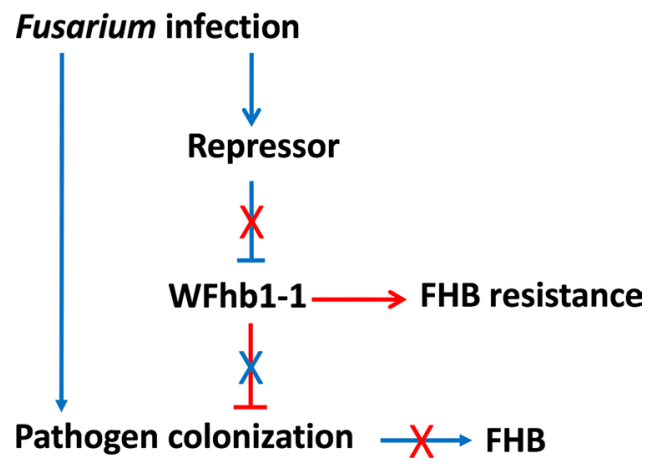

Figure 8. An illustration showing the hypothesis on how WFhb1-1 suppresses infecting pathogen leading to FHB resistance and how the infecting pathogen suppresses WFhb1-1 expression to gain colonization and thus develop FHB on the host plant. Arrowhead lines indicate promotion, T-headed lines denotes suppression, $\mathrm{X}$ means interruption, blue lines/letter lead to FHB development and red line/letter results in FHB resistance.

Therefore, our findings show that WFhb1-1 is a major FHB-resistance gene in wheat that can significantly reduce both FHB severity and DON accumulation even under high disease pressure. Wang et al. ${ }^{48}$ reported that WFhb11 not only confers FHB resistance but also shows resistance against Fusarium root rot (FRR) by preventing the pathogen from spreading in the infected wheat plants. It seems that WFhb1-1 works in the whole plant to protect it against Fusarium-caused diseases.

Our data show that $P$. pastoris-expressed WFhb1-1 protein can inhibit growth of both $P$. pastoris and $F$. graminearum in culture (Fig. 2D-G). These results suggest that WFhb1-1 is most likely an antifungal protein and probably functions to inhibit F. graminearum colonization in planta. Since our sequence analysis did not reveal any DON detoxification domain in WFhb1-1, the observed reduction in DON content in the kernels of the WFhb1-1-overexpressing NIL-S plants (Fig. 6C; Supplementary Table S2) is probably due to a reduced fungal population on the plants by WFhb1-1's antifungal activity, not due to DON detoxification by WFhb1-1. It will be interesting to know if WFhb1-1 has an even broader role in protecting wheat against more fungal diseases other than FHB and FRR.

Our study has confirmed our previous conclusion that F. graminearum can suppress WFhb1-1 expression in the early hours of FHB pathogenesis (Fig. 3; Supplementary Table S1). This suppression may be a key step in FHB resistance. In a previous study based on quantitative proteomic analysis of the same Qfhb1 NILs, we found that FHB may result from a pathogen-promoted hypersensitive reaction (HSR) by the infected host cell, and Qfhb1 largely functions to either alleviate HSR or to manipulate the host cells to not respond to the pathogen promotion ${ }^{43}$. The results from the present study suggests that the reduction of the pathogen population on the plant by WFhb1-1 might lead to significantly less infected host cells and thus significantly less HSR promotion in the host tissues by the pathogen. Therefore, as illustrated in Fig. 8, here we hypothesize a pathogen-host interaction model: WFhb1-1 inhibits the growth of F. graminearum to prevent FHB development, the pathogen has developed an ability during the host-pathogen co-evolution to overcome WFhb1-1's inhibition by suppressing its transcription during the initial stage of infection which leads to FHB development, and wheat then develops a currently unknown way to avoid the pathogen's suppression resulting in resistance to FHB again. In this model, we hypothesize that the pathogen may either produce and then deliver a WFhb1-1 suppressor into the host, or it may negatively regulate an indigenous host suppressor. In the first scenario, WFhb1-1 may work alone in contribution to FHB resistance. The second scenario actually fits to the multi-gene model of Qfhb1-confered FHB resistance proposed by Gao et al. ${ }^{11}$, Schweiger et al. ${ }^{16}$ and Rawat et al. ${ }^{17}$. The pathogen's inability to suppress the WFhb1-1 transcription from BSMV:W suggests that the suppression may target at the native WFhb1-1 promotor or another regulation site but not WFhb1-1 transcript itself. More research is needed to test this hypothesis and answer the question how FHB-resistant wheat genotypes escape this suppression.

Two recent publications have associated TaHRC with the Qfhb1-confered FHB resistance ${ }^{19,20}$. Although the resistance mechanisms reported in the two reports are controversial, both concluded that TaHRC is an FHB-susceptibility gene and a deletion in this gene has caused the loss of the FHB susceptibility. Su et al. ${ }^{19}$ reported that $\mathrm{TaHRC}$ is a nuclear protein with an unknown biological function. Therefore, it could be a regulator to other functional genic components of $Q f h b 1$. In the recent study, we have found that knocking TaHRC out in FHB-susceptible wheat cultivar Bobwhite by CRISPR/Cas9 gene editing also causes upregulation of WFhb1-1. This result indicates that TaHRC might negatively regulate WFhb1-1 expression. Therefore, it is highly probable that TaHRC is the WFhb1-1-suppressor in our model presented in Fig. 8, and in the cases reported by Su et al. ${ }^{19}$ or Li et al. ${ }^{20}$, a deletion in TaHRC causes the loss of its suppression of WFhb1-1, which results in a functional WFhb11 during the pathogen infection and thus FHB resistance. Further researches should reveal how TaHRC regulates WFhb1-1 and whether the pathogen indeed interacts with TaHRC to develop FHB.

As described by Zhuang et al. ${ }^{15}$, we previously identified WFhb1-cl as a candidate of the functional genic component of $Q f h b 1$ on the basis of the following evidences: (1) WFhb1-1 was differentially expressed between NIL-S and NIL-R early in the pathogenesis ${ }^{15,38}$, which determines the FHB resistance or susceptibility in the NILs; (2) out of the 406 FHB-related genes investigated, WFhb1-1 was the only one whose expression was significantly associated with Qfhb1 by eQTL mapping ${ }^{15}$, which suggests that WFhb1-1 either physically locates in the QTL or is functionally controlled by a gene in this QTL; and (3) nullisomic-tetrasomic analysis has physically mapped it 
on chromosome arm $3 \mathrm{BS}^{15}$. These arguments have been strengthened by the following findings from the present study: First, suppression of WFhb1-1 accumulation at the early stage of FHB pathogenesis was observed in NIL-S but not in NIL-R (Fig. 3); secondly, WFhb1-1 has been found to have antifungal ability (Fig. 2), thus, WFhb1I's role in FHB resistance seems to be an inhibitor to the pathogen growth in planta, which is well-aligned with Qfhbl's function of conferring Type II resistance; and thirdly, FHB-susceptible NIL-S plants can be made as resistant as NIL-R plants are by simply overexpressing WFhb1-1 in them, while silencing WFhb1-1 in Sumai 3 resulted in complete compromise of FHB resistances (Fig. 4).

However, the present study still does not give a conclusive answer to the question whether WFhb1-1 is indeed physically located in the Qfhb1 interval or not. This is because WFhb1-1 has been in silico mapped to a place between markers Xsts9-2 and Xsts65-3 in the 3BS pseudomolecule (Fig. 1B), which is found to be within the Qfhb1 interval defined by some ${ }^{15,23,29}$ but outside the interval defined by others ${ }^{16,17,31}$. Particularly, WFhb1-1 is not among the genes in the QTL interval reported by Schweiger et al. ${ }^{16}$ or by Rawat et al. ${ }^{17}$. This controversy could be at least partially due to sequence diversity in the Qfhb1-containing region among wheat cultivars. In fact, Schweiger et al. ${ }^{16}$ reported a high dissimilarity between Sumai 3 and Chinese Spring in the core region of $Q f h b 1$ that hosts the FHB marker UMN10 and the three published Qfhbl candidate genes: GDSL, PFT, and HRC. Furthermore, several landmark markers of this QTL have inconsistent positions on 3BS among the wheat accessions used in different mapping studies, indicating occurrence of inversions among these wheat accessions. For example, marker Xbarc147 is moved from the proximal side of marker Xgwm533 in the Sumai 3/Stoa map by Liu et al ${ }^{49}$ to the distal side of Xgwm533 in the Wangshuibai/Wheaton map by Yu et al. ${ }^{50}$ and the CS-SM3-3B/ Annong8455 map by Zhou et al..$^{0}$; Xgwm493 is at the distal side of Xumn10 in the Chinese Spring 3B pseudomolecule (Fig. 1B) but at its proximal side in the Sumai 3/Stoa map by Liu et al. ${ }^{49}$. Also, marker Xbarc147 is at the distal side of WFhb1-1 in our map ${ }^{15}$ and the Qfhb1 interval in the Sumai 3/Stoa map by Liu et al. ${ }^{49}$ but in the middle of $Q f h b 1$ in the Wangshuibai/Wheaton map by Yu et al. ${ }^{50}$. Furthermore, $P F T$, the proposed candidate gene of $Q f h b 1$ by Rawat et al. ${ }^{17}$, was in fact mapped outside the QTL in FHB-resistant Wangshuibai and Sumai 3 according to Li et al. ${ }^{20}$. Therefore, it is possible that WFhb1-1 and its regulator gene are both in the Qfhb1 interval in some wheat lines but, in others, only the regulator is in the QTL with WFhb1-1 itself flanking outside. Nevertheless, our data indicate that, being a genic component of $Q f h b 1$ or not, WFhb1-1 is likely a key contributor to FHB resistance conferred by $Q f h b 1$ in wheat.

In summary, findings from this study demonstrate that WFhb1-1 is likely a key FHB-resistance gene in wheat with potential antifungal function, and it most likely is a functional component of $Q f h b 1$ because $Q f h b 1$ cannot confer FHB resistance without WFhb1-1. Our data show that wheat needs a normal WFhb1-1 expression at the initial stage of FHB pathogenesis to develop FHB resistance, while the pathogen needs to suppress WFhb1-1 expression to colonize and thus cause FHB in wheat. Understanding how the pathogen suppresses WFhb1-1 transcription will greatly help to our understanding of FHB pathogenesis and thus the control of FHB epidemics.

\section{Materials and Methods}

The formulas of the buffers and media used in this study are listed in Supplementary Document S3. All the sequences of the adaptors and PCR primers used in this study are listed in Table 1.

Plant materials. Bread wheat cultivar Sumai 3 (FHB-resistant), landrace Y1193-06 (FHB-susceptible), a pair of Qfhb1 NILs 206-1-1-2 (NIL-R) and 260-1-1-4 (NIL-S), Bobwhite (FHB-susceptible) and its CRISPR/ Cas9-edited TaHRC-knockout mutant were used in this study. The NILs were developed and kindly provided by Dr. James Anderson's lab at University of Minnesota. The TaHRC knockout Bobwhite mutant was kindly provided by Dr. Guihua Bai of USDA-ARS/Kansas State University. For each experiment, at least 10 plants per line per repeat per treatment were grown in pots filled with Miracle-Growth Potting Mix in a greenhouse or a

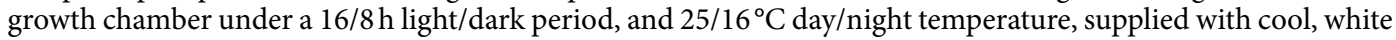
fluorescent lamps.

Rapid Amplification of CDNA ends (RACE) and Gene Cloning. RACE experiments were conducted using total RNA isolated from Sumai 3 and the 5'/3' RACE Kit, $2^{\text {nd }}$ Generation (Roche Life Science). Gene-specific primers were designed according to the EST sequence (GenBank \#: CA640991) that probe TaAffx.111425.2.S1_ at on the Affymetrix Wheat Genome GeneChip was based on. The $5^{\prime}$ and $3^{\prime}$ RACE products were cloned and sequenced separately. The full-length cDNA was assembled by merging the $5^{\prime}$-and the $3^{\prime}$-amplicons from the RACEs, and then confirmed by PCR cloning of the whole sequence from Sumai 3 . The cloned sequence was also compared with the publicly available chromosome arm 3BS pseudomolecule reference sequence of wheat cultivar Chinese Spring (now part of wheat reference genomic sequence IWGSC RefSeq v1.0.).

Genomic sequences of the gene of interest were PCR-cloned from Sumai 3 genomic DNA with primers designed based on the 3BS pseudomolecule sequence. Again, the PCR products of interest were cloned, sequenced and validated by comparing them to the relevant sequences in the $3 \mathrm{BS}$ pseudomolecule. Putative promoter sequences were predicted using TSSP and RegSite PlantProm DB (http://www.softberry.com/berry. phtml?topic=promoter) and Neural Network Promoter Prediction (http://www.fruitfly.org/seq_tools/promoter. html). Open reading frames were predicted by ORFfinder at NCBI.

Prediction of protein property. Protein properties were predicted with Phobius, a combined transmembrane topology and signal peptide predictor at http://phobius.binf.ku.dk using the normal prediction function ${ }^{51}$. Analysis of conserved protein domains was done using quick scan mode of ScanProsite (http://prosite.expasy.org).

Protein expression in Pichia pastoris and in vitro inhibition assay of $\boldsymbol{F}$. graminearum. Antibody design. Primary antibodies were raised in rabbit against the peptides P Q R P P A V G P F P W E and Q Q P P A S P R S G S G F P, respectively, which were selected from the putative protein sequence of WFhb1-1 (Fig. 1A) 


\begin{tabular}{|l|l|}
\hline Name & Sequence \\
\hline WFhb1-1 silencing insert Forward & TGTGCTCTGCTTTCCTGCTG \\
\hline WFhb1-1 silencing insert Reverse & CCCAGCATACAGTTGAAACG \\
\hline WFhb1-1 expression Forward & CTGAGCGGCTGCTGTGCTGA \\
\hline WFhb1-1 expression Reverse & ATATCAGATCTGGCAGTGCCCCA \\
\hline BSMV:T stability Forward & TGATGATTCTTCTTCCGTTGC \\
\hline BSMV:T stability Reverse & GTTTCCAATTCAGGCATCGT \\
\hline pTMH Forward & TGCTACATCCATACTCCATCCTTCCC \\
\hline pTMH Reverse & AGCTGACATCGACACCAACGATCT \\
\hline UBC Forward & TCCCCTTACTCTGGCGGTGTC \\
\hline UBC Reverse & TTGGGGTGGTAGATGCGTGTAGT \\
\hline WFhb1-1-ORF-Forward & CACGCAGTTCCCCTTCTG \\
\hline WFhb1-1-ORF-Reverse & GAGCAGCAGCAAAGCAGAG \\
\hline 5'AOX & GACTGGTTCCAATTGACAAGC \\
\hline $3^{\prime}$ AOX & GCAAATGGCATTCTGACATCC \\
\hline Gamma-1-Forward & CGCAATACGTAAGTCCGTAGC \\
\hline Gamma-1-Reverse & GATGGGCACCATCAGATTT \\
\hline Wheat $\beta$-actin Forward & AAATCTGGCATCACACTTTCTAC \\
\hline Wheat $\beta$-actin Reverse & GTCTCAAACATAATCTGGGTCATC \\
\hline HRC-qPCR Forward & CAGCAGAGTTCACACGATGA \\
\hline HRC-qPCR Reverse & GGTGAGCCAGACAAGATGAA \\
\hline His Forward & CAAGTACAGGCTTCAGAATCCA \\
\hline His Reverse & GCAACTCGTGTAAGTTGTTAAAA \\
\hline PFT Forward & GGATCTGGGCTGATTCAACT \\
\hline PFT Reverse & TTTCGCAGAGCAATGAAGTC \\
\hline GDSL Forward & TCAACAGGAGCCAGTTTGTC \\
\hline GDSL Reverse & GATGTCCAAGGTGTAAAGCG \\
\hline & \\
\hline
\end{tabular}

Table 1. Sequences of adaptors and PCR primers used in this study.

following predictive analysis of protein folding. The antibodies were produced at GenScript USA Inc. The primary antibody against the protein motif P Q R P P A V G P F P W E was named PA-1, and the primary antibody against the protein motif Q Q P P A S P R S G S G F P was named PA-2.

Construction of expression vector. WFhb1-1's ORF was synthesized according to the cloned gene sequence with an $E c o R$ I site added at the $5^{\prime}$ end and a Xho I site added at the $3^{\prime}$ end. The synthesized foreign gene expression insert was put into $p U C 57$ and cloned into Escherichia coli JM 109 by heat shock method. Briefly, $1 \mu \mathrm{g} p U C 57$ insert plasmid was added to $50 \mu \mathrm{l} \mathrm{E}$. coli cells. The mixture was incubated on ice for $20 \mathrm{~min}$, heat-shocked at $42^{\circ} \mathrm{C}$ for $50 \mathrm{~s}$ and immediately kept on ice for $2 \mathrm{~min}$. Then, $950 \mu \mathrm{l} \mathrm{SOC}$ medium was added into mixture followed by $\sim 1.5 \mathrm{~h}$ incubation at $37^{\circ} \mathrm{C}$ with shaking at $\sim 150 \mathrm{rpm}$. The culture $(50 \mu \mathrm{l}$ or $100 \mu \mathrm{l})$ was spread on LB agar plates (with ampicillin $100 \mu \mathrm{g}$ per $\mathrm{ml}$ ) in duplicate. The plates were incubated at $37^{\circ} \mathrm{C}$ overnight and plasmid DNA was extracted from developed colonies for further use.

EasySelect ${ }^{\mathrm{TM}}$ Pichia Expression Kit (ThermoFisher Cat \# K174001) was used to express the protein of interest in $P$. pastoris yeast. First, $p P I C Z A$ was used to make the yeast expression vector. Briefly, the $p U C 57-$ foreign gene plasmid was digested with EcoR I and Xho I enzymes to release the expression insert, which was then ligated between the pre-cut EcoR I and Xho I sites in PPICZA using the T4 DNA ligase in 2X rapid ligation buffer from Promega. After the desired orientation of the insert in $P P I C Z A$-foreign gene was confirmed, nearly $10 \mu \mathrm{g}$ of pPICZA-WFhb1-1 was linearized with Sac I. The linearization was confirmed by running the digested and undigested plasmid parallelly in $1 \%$ agarose gel. For preparing competent cells of $P$. pastoris strain X33, the yeast cells were cultured in $5 \mathrm{~mL} \mathrm{YPD} \mathrm{(yeast} \mathrm{extract} \mathrm{peptone} \mathrm{dextrose)} \mathrm{medium} \mathrm{in} \mathrm{a} 50$-mL conical flask at $29^{\circ} \mathrm{C}$ overnight. Fifty milliliters of fresh YPD medium in a $250-\mathrm{mL}$ conical flask was inoculated with $0.5 \mathrm{~mL}$ of the overnight culture and was again incubated at $29^{\circ} \mathrm{C}$ overnight. Next day, the culture was centrifuged at $1500 \times \mathrm{g}$ for $5 \mathrm{~min}$ at $4^{\circ} \mathrm{C}$ to harvest the cells. The supernatant was discarded, and the cells were resuspended in $50 \mathrm{~mL}$ of ice-cold, sterile water. The cells were again centrifuged as above and resuspended in $25 \mathrm{~mL}$ of ice-cold, sterile water. The cells were centrifuged again with the same speed and dissolved in $2 \mathrm{~mL}$ of ice-cold, sterile $1 \mathrm{M}$ sorbitol. The cells were centrifuged one more time as above and dissolved in $0.5 \mathrm{~mL}$ of ice-cold, sterile $1 \mathrm{M}$ sorbitol. Now the P. pastoris cells are ready for transformation by electroporation.

For transformation by electroporation, about $10 \mu \mathrm{g}$ of the Sac I-linearized $p P I C Z A$ expression vector was mixed with $80 \mu \mathrm{L}$ of the $P$. pastoris competent cells and the mixture was transferred into an ice-cold 2-mm electroporation cuvette. The cuvette with the mixture was incubated on ice for 5 minutes. The cuvette was then put in BioRad GenePulsar X cell and pulsed once with pre-set protocol for P. pastoris with the parameters as follows: $2000 \mathrm{~V}, 25 \mu \mathrm{F}, 200$ ' $\Omega$ and $2 \mathrm{~mm}$. Immediately after the pulsing, $1 \mathrm{~mL}$ of ice-cold, sterile $1 \mathrm{M}$ sorbitol was added in the cuvette, and the cuvette content was transferred into a sterile $15 \mathrm{ml}$ tube. The tube was incubated at $29^{\circ} \mathrm{C}$ 
without shaking for 1.5 hours. Then, the incubated culture in the tube was spread on YPD agar plates containing $100 \mu \mathrm{g} / \mathrm{mL}$ zeocin. Different volumes $(10,20,50,100$, and $200 \mu \mathrm{L})$ of the culture were spread on separate YPD plates with zeocin. The plates were then incubated at $29^{\circ} \mathrm{C}$ until the colonies formed (about a week). Then, 10 colonies were picked and the X33:T expression strain was further confirmed by its Mut + phenotype following the protocol from Invitrogen (https://tools.thermofisher.com/content/sfs/manuals/easyselect_man.pdf). X33:T was also confirmed by PCR using WFhb1-1-ORF forward \& reverse, 5'AOX and 3'AOX primers. X33 was also transformed with $p P I C Z A-w t$ (the wildtype $p P I C Z A$ plasmid without the expression insert) to form the background control strain X33:00, following the same protocol as explained above.

Protein expression in the P. pastoris expression system. For the protein expression experiments, a single colony of X33:T or X33:00 was cultured in $25 \mathrm{~mL}$ of MGY (minimal glycerol yeast) medium in a 250-mL conical flask and incubated at $29^{\circ} \mathrm{C}$ for $16-18 \mathrm{~h}$ with shaking $(250 \mathrm{rpm})$. To induce foreign protein expression in P. pastoris, cells were harvested by centrifuging at $3000 \times \mathrm{g}$ for $5 \mathrm{~min}$ at RT and the supernatant was discarded. The cell pellet was then resuspended in $25 \mathrm{~mL}$ of MM (minimal methanol) medium in a $250-\mathrm{mL}$ conical flask, covered with two-layer sterile cheesecloth, and continued to incubate at $29^{\circ} \mathrm{C}$ in the shaker incubator $(250 \mathrm{rpm})$. Methanol was added to a final concentration of $0.5 \%$ every 24 hours to maintain the expression induction. At each time points (Day0, Day1, Day2, Day3, and Day4), $1 \mathrm{~mL}$ of the expression culture was collected into a $1.5 \mathrm{~mL}$ microcentrifuge tube. This sample is used to analyze the growth kinetics by counting the cells and to extract the total protein.

Pichia pastoris growth kinetics. P. pastoris growth kinetics were studied by counting the number of cells per milliliter in the expression culture in Day0, Day1, Day2, Day3 and Day4 starting from the time when methanol was first added into the MM medium (Day0). This was done by first diluting $10 \mu \mathrm{L}$ of the culture from each time point with $990 \mu \mathrm{L}$ of sterile water to make a 100 -fold dilution. The diluted culture samples were then microscopically observed on a hemocytometer to count the cells in the four squares with $0.1 \mathrm{~mm}^{3}$ per square. The cell numbers in the four squares were averaged and multiplied with the dilution factor to get the final count as "cells /mL". The budding cells attached together were counted as a single cell. This experiment was repeated for three times.

Protein extraction and quantification. Total proteins were extracted from both X33:T and X33:00. One milliliter of the expression culture sample was collected into a $1.5 \mathrm{~mL}$ microcentrifuge tube at each time point and centrifuged at maximum speed in a tabletop centrifuge for 2 minutes at room temperature. The supernatant was transferred into a new $1.5 \mathrm{~mL}$ tube. Both the supernatant and the pellet were snap-frozen in liquid nitrogen and stored at $-80^{\circ} \mathrm{C}$ until further processed. Total protein concentration of this supernatant was measured at $562 \mathrm{~nm}$ absorbance using Pierce BCA Protein Assay Kit from Thermo-Scientific, and final concentration of $1 \mu \mathrm{g} / \mu \mathrm{L}$ was maintained for all the samples by adding adequate MM medium.

For the extraction of total protein from pellet sample, each pellet was resuspended in $100 \mu \mathrm{L}$ breaking buffer. Equal volume of acid-washed glass beads (size $0.5 \mathrm{~mm}$ ) was then added into the pellet suspension and vortexed for $30 \mathrm{~s}$. The mixture was incubated on ice for $30 \mathrm{~s}$ and then vortexed before another $30 \mathrm{~s}$ incubation. This cycle was repeated for eight times. After the final vortexing, the mixture was centrifuged at maximum speed for $10 \mathrm{~min}$ at $4{ }^{\circ} \mathrm{C}$. The clear supernatant was then transferred to a fresh $1.5 \mathrm{~mL}$ microcentrifuge tube. Total protein concentration was measured at $562 \mathrm{~nm}$ absorbance using Pierce BCA Protein Assay Kit from Thermo-Scientific, and $1 \mu \mathrm{g} / \mu \mathrm{L}$ final concentration was achieved by adding breaking buffer.

For extraction of total protein from wheat spikelets, each sample harvested at a desired time point were ground in $1.5 \mathrm{~mL}$ tubes with plastic pestle in liquid nitrogen until the fine powder was obtained. After grinding the sample, $200 \mu \mathrm{L}$ of breaking buffer was added in each tube. Vortexing was done with intermittent incubation of tubes on ice (vortexing for $30 \mathrm{~s}$ followed by incubation on ice for $30 \mathrm{~s}$ for total $8 \mathrm{cycles}$ ). Then, the tubes were centrifuged at maximum speed at $4{ }^{\circ} \mathrm{C}$ for $10 \mathrm{~min}$, and the clear supernatant was transferred into new micro-centrifuge tubes. Protein concentration was measured with $280 \mathrm{~nm}$ absorbance, and the concentration was also confirmed using Pierce $^{\mathrm{TM}}$ BCA Protein Assay Kit (ThermoFisher Cat \# 23250). Additional breaking buffer, if needed, was added to make the final concentration of each protein sample $1 \mu \mathrm{g} / \mu \mathrm{L}$. These extracted protein samples were stored at $-80^{\circ} \mathrm{C}$ until further used.

In vitro inhibition assay of F. graminearum with the yeast-produced protein. The total protein in breaking buffer in a $1.5-\mathrm{mL}$ microcentrifuge tube was precipitated by adding four times (in volume) of cold acetone (stored at $-20^{\circ} \mathrm{C}$ ) into the tube. The tube was then vortexed and incubated for $1 \mathrm{~h}$ at $-20^{\circ} \mathrm{C}$. Then, the tube was centrifuged for 10 minutes at $15000 \times \mathrm{g}$, and the supernatant was carefully discarded. The protein pellet was air-dried by leaving the tube lid open at RT for about $30 \mathrm{~min}$. The dried pellet was then dissolved in adequative amount of sterile distilled water to make the total protein solutions of desired concentrations, which was measured by the standard BSA protein assay. Series of total protein concentrations $(20,50,100,500,600,700 \mu \mathrm{g} / \mathrm{mL})$ in each volume were made and used in the inhibition assay. To produce a larger amount of total protein, the scale was increased by 50 folds.

To assay if the yeast-produced protein can inhibit F. graminearum growth, about $10,000 \mathrm{~F}$. graminearum conidia were grown in $100 \mu \mathrm{L}$ of potato dextrose broth (PDB) per well in a 48 -well cell culture plate. Total protein extracted from either X33:T or X33:00 cultures in a concentration of 20,50, 100, 500, 600 or $700 \mu \mathrm{g} / \mathrm{mL}$ was added into three parallel wells, and sterile water was used as blank control. Each treatment was repeated three times. Growth of F. graminearum in each well was visually assessed in two weeks after the treatment started, and high resolution pictures for plates were taken. The intensity of the fungal growth in each well was calculated and analyzed by using ImageJ software ${ }^{52}$. In brief, equal area of each wells was selected and the intensity of the selected area was calculated in ImageJ for each wells with fungal growth and the statistical analysis was done. 
Polyacrylamide gel electrophoresis and Western blotting. For SDS-PAGE, $25 \mu \mathrm{g}$ of total protein was loaded in each well of BioRad pre-casted mini polyacrylamide gels. Precision plus protein standard (BioRad) was used to estimate the protein mass. The gel was run for about $1 \mathrm{~h}$ with $30 \mathrm{~mA}$ constant current in a Bio-Rad mini PROTEAN tetra cell, and then was either processed for Sypro-Ruby staining or for the Western blotting.

For Sypro-Ruby staining, the gel was first fixed in fixing solution (50\% methanol and $10 \%$ acetic acid in distilled water) for $1 \mathrm{~h}$. Then, the fixing solution was discarded, and the gel was soaked in $50 \mathrm{~mL}$ Sypro-Ruby protein gel stain solution for $2 \mathrm{~h}$ with gentle agitation and protection from light. Then the gel was transferred into a clean tray and washed with wash solution (10\% methanol and $7 \%$ acetic acid in distilled water) with gentle shaking for $15 \mathrm{~min}$. The gel is then visualized and recorded under UV.

For Western blotting, after the SDS-PAGE was run, the total protein on the gel was transferred onto a nitrocellulose membrane using iBlot from Invitrogen. Then, the membrane was incubated in buffer TBST with 5\% dry milk for $1 \mathrm{~h}$ with gentle shaking for blocking. The membrane was then washed three times with buffer TBST having $0.5 \%$ dry milk ( 10 minutes each time with gentle shaking). Then the membrane was incubated in primary antibody solution diluted to $1: 10,000$ in TBST with $0.5 \%$ dry milk for $1 \mathrm{~h}$ at RT with gentle shaking. Then the membrane was washed for 3 times with TBST having $0.5 \%$ milk as described above. LICOR IRdye $800 \mathrm{CW}$ goat anti-rabbit antibodies were used for secondary binding and visualization. Same dilution as primary antibodies $(1: 10,000)$ was used in TBST with $0.5 \%$ dry milk, and the membrane was incubated at RT for $1 \mathrm{~h}$ with gentle shaking. The membrane was then washed again 3 times as described above and visualized in LICOR Odyssey Fc under $800 \mathrm{~nm}$ absorbance with exposure time of 2 milliseconds.

Western Blots of total wheat protein were digitized for fluorescence signal strength with LICOR Odyssey Fc and analyzed. At least three bio-repeats per treatment and three technical repeats per bio-repeat were performed. For each NIL, the signal data were compared between the treatment and the mock control for each time point and the relative change were then normalized by taking the value at $0 \mathrm{hpfi}$ (hours post Fusarium inoculation) off (Supplementary Table S1).

Constructing overexpression and knockdown vectors. A barley stripe mosaic virus (BSMV)-based virus-induced gene overexpression (VOX)/silencing (VIGS) system was used to transiently overexpress or silence the gene of interest. The BSMV vectors used were kindly provided by Dr. Li Huang of Montana State University. The BSMV $\gamma$ vector ( $\gamma$ PCR vector) was modified by Huang's group with two Xcm I restriction sites inserted. We further modified it by adding an $E c o R$ I site between the two $X \mathrm{~cm}$ I sites. To construct the overexpression vector BSMV:W, WFhb1-1's ORF (384 bp) with a EcoR I site at each end was synthesized by GenScript. BSMV:W was then constructed by digesting the $\gamma$ PCR vector and the synthesized ORF with EcoR I, and mixed then together to have the ORF inserted in the EcoR I site. The desired orientation of the ORF was confirmed with PCR using WFhb1-1-ORF forward/Gamma-1 reverse primers.

To produce RNAi silencing BSMV vector VIGS:T, TA cloning site was first produced from the $\gamma$ PCR vector by digesting it with XcmI. A fragment of 289-bp complementary to the corresponding WFhb1-1 coding sequence was amplified by PCR from Sumai 3 with WFhb1-1 silencing primer set (Table 1) and ligated into the $\gamma$ PCR vector. Both the VOX and VIGS vectors were confirmed by sequencing.

In vitro transcription of viral RNA and plant inoculation. The three BSMV RNA chromosomes were reverse transcribed from the corresponding BSMV vectors following the protocol provided by Dr. Li Huang of Montana State University. RNA quality was assessed on $1 \%$ agarose gel. Virus inoculation was done by following the previously described inoculation procedures ${ }^{53,54}$. Briefly, a mixture of the three viral RNAs in viral inoculation buffer FES was manually rubbed into plant tissue.

Viral inoculation was carried out with either the VOX vector BSMV:W, the VIGS vector BSMV:T, the empty BSMV control vector BSMV:00 or the viral inoculation buffer FES alone on at least 10 plants per line per treatment. The inoculation was done either on a spike at shooting stage as soon as three fourths of the spike was emerged, on a 10-day old leaf, or on a flag leaf at the booting stage when the flag leaf was fully expanded.

Fungal inoculum preparation, inoculation, sampling and disease evaluation. F. graminearum isolate Fg4, collected from Watertown, SD was used in this study to induce FHB. F. graminearum was cultured on potato-dextrose-agar (PDA) medium for a week, and then spores were collected for plant inoculation. Procedures used for wheat spike inoculation as previously described ${ }^{34}$. Briefly, F. graminearum spores were washed from PDA plates using sterile water and then filtered through four layers of sterile cheesecloth. The concentration of conidia was counted using a hemocytometer and adjusted with sterile water to about 100,000 conidia $/ \mathrm{mL}$. The spikelet was challenged with $10 \mu \mathrm{L}$ of water-suspension of F. graminearum conidia or sterile water alone (as a mock control) at the stage when intensive yellow color of anthers was observed. For each treated spike, the two first-flowering spikelets were inoculated to introduce a disease pressure at the level that an FHB-resistant genotype will be maximumly diseased at $28 \mathrm{dpfi}$. The inoculated spikes were immediately covered with plastic zip-lock bags with a wet cotton ball inside for $72 \mathrm{~h}$ to maintain the needed humidity and temperature inside the bags (greenhouse effect) to facilitate disease establishment. The length between the BSMV and the F. graminearum inoculations was optimized for both leaf and spike inoculations by test inoculation of the two at various time intervals until the maximum effect of overexpression or knockdown of WFhb1-1 was reached, usually at $4 \sim 7$ dpvi when yellow pollens emerged out of the first pair of spikelets.

For disease evaluation, FDR was calculated as percentage of diseased rachides of all rachides per spike and FDK was calculated as percentage of diseased kernels of all harvested kernels per spike. FDR were averaged per time-point per treatment per experiment at 7, 14/15, 21 and 28 dpfi, respectively. DON content in the harvested 
kernels per spike was also measured by sending the harvested kernels to the DON testing lab at University of Minnesota and analyzed for each treatment in our lab.

Quantitative Real-time PCR (RT-qPCR). Total RNA was extracted from leaf, spike or spikelet samples with TRIZOL (Invitrogen) following the manufacturer's instructions. RNA quality was tested using $0.8 \%$ or $1 \%$ agarose gel and quantified using a NanoDrop ND-1000 UV-Vis Spectrophotometer (Wilmington, DE). For each treatment, three to four biological replications were conducted. For reverse transcription, $\sim 500$ ng DNase I-treated total RNA was used for cDNA synthesis using GoScript ${ }^{\mathrm{TM}}$ Reverse Transcriptase system (Promega) with oligo $(\mathrm{dT})_{15}$ primer. For VOX and VIGS experiments, if relevant, the presence of BSMV viral genome in the cDNA samples was confirmed before RT-qPCR was conducted. The RT-qPCR was conducted on a Smart Cycler II (Cepheid) or QuantStudio 6 Flex (Applied Biosystems). Briefly, 2X dilutions were made for reverse transcription products, and $1 \mu \mathrm{L}$ diluted cDNA/20 $\mu \mathrm{l}$ reaction was carried out using SYBR green I master Mix with $2 \mathrm{~min}$ at $95^{\circ} \mathrm{C}, 45$ cycles of $20 \mathrm{~s}$ at $95^{\circ} \mathrm{C}, 30 \mathrm{~s}$ at melting temperature, and $30 \mathrm{~s}$ at $72{ }^{\circ} \mathrm{C}$, and then $5 \mathrm{~min}$ at $72^{\circ} \mathrm{C}$. Wheat $\beta$-actin gene was used as an internal control to normalize the $C t$ value. For each sample, three technical replications were conducted. Fold changes were calculated with the $2^{-\Delta \Delta C t}$ method $^{55}$.

Statistical analyses. Tukey's multiple comparisons of means in $R$ software package was used to compare FDR and FDK values of each treatment at the 95\% family-wise confidence level. We also conducted student $t$-test and one-way ANOVA analysis for these data. The association between transcript abundance of a gene of interest and FDR in the gene silencing experiment was measured using Pearson's product-moment correlation analysis in $R$ software package.

Received: 10 November 2019; Accepted: 17 April 2020;

Published online: 08 May 2020

\section{References}

1. Windels, C. E. Economic and social impacts of Fusarium head blight: changing farms and rural communities in the Northern Great Plains. Phytopathology 90, 17-21 (2000).

2. Wilson, W., Dahi, B. \& Nganje, W. Economic costs of Fusarium head blight, scab and deoxynivalenol. World Mycotoxin J. 11, 291-302 (2018)

3. Desjardins, A. E. \& Hohn, T. M. Mycotoxins in plant pathogenesis. Mol. Plant-Microbe 10, 147-152 (1997).

4. McMullen, M., Jones, R. \& Gallenberg, D. Scab of wheat and barley: a re-emerging disease of devastating impact. Plant Dis. 81, 1340-1348 (1997).

5. Snijders, C. Fusarium head blight and mycotoxin contamination of wheat, a review. Netherlands J. Plant Pathol. 96, 187-198 (1990).

6. Dahl, B. \& Wilson, W. W. Risk premium due to Fusarium head blight in wheat and barley. Agr. Syst. 162, 145-153 (2018).

7. Pestka, J. J. Deoxynivalenol: mechanisms of action, human exposure, and toxicological relevance. Arch. Toxicol. 84, 663-679 (2010).

8. Schroeder, H. \& Christensen, J. Factors affecting resistance of wheat to scab caused by Gibberella zeae. Phytopathology 53, 831-838 (1963).

9. Mesterhazy, A. Types and components of resistance to Fusarium head blight of wheat. Plant Breed. 114, 377-386 (1995).

10. Foroud, N. et al. Differential transcriptome analyses of three wheat genotypes reveal different host response pathways associated with Fusarium head blight and trichothecene resistance. Plant. Pathol. 61, 296-314 (2012)

11. Gao, L., Chen, F., Zhou, L. \& Lu, W. Genetic analysis of resistance to wheat scab (Fusarium graminearum Schw) in Wangshuibai. J. Triticeae Crops 25, 5-9 (2005).

12. Ginkel, M. V., Schaar, W. V. D., Zhuping, Y. \& Rajaram, S. Inheritance of resistance to scab in two wheat cultivars from Brazil and China. Plant Dis. 80, 863-867 (1996).

13. Buerstmayr, H., Ban, T. \& Anderson, J. A. QTL mapping and marker-assisted selection for Fusarium head blight resistance in wheat: a review. Plant Breed. 128, 1-26 (2009).

14. Liu, S. X. et al. Toward positional cloning of Fhb1, a major QTL for Fusarium head blight resistance in wheat. Cereal Res. Comm. 36, 195-201 (2008)

15. Zhuang, Y., Gala, A. \& Yen, Y. Identification of functional genic components of major Fusarium head blight resistance quantitative trait loci in wheat cultivar Sumai 3. Mol. Plant-Microbe Interact. 26, 442-450 (2013).

16. Schweiger, W. et al. Suppressed recombination and unique candidate genes in the divergent haplotype encoding Fhb1, a major Fusarium head blight resistance locus in wheat. Theor. Appl. Genet. 129, 1607-1623 (2016).

17. Rawat, N. et al. Wheat Fhb1 encodes a chimeric lectin with agglutinin domains and a pore-forming toxin-like domain conferring resistance to Fusarium head blight. Nat. Genet. 48, 1576-1580 (2016).

18. Giancaspro, A. et al. Cell wall features transferred from common into durum wheat to improve Fusarium Head Blight resistance. Plant Sci. 274, 121-128 (2018).

19. Su, Z. et al. A deletion mutation in TaHRC confers Fhb1 resistance to Fusarium head blight in wheat. Nat. Genet. 51, 1099-1105 (2019).

20. Li, G. et al. Mutation of a histidine-rich calcium-binding-protein gene in wheat confers resistance to Fusarium head blight. Nat. Genet. 51, 1106-1112 (2019).

21. Gadaleta, A., Colasuonno, P., Giove, S. L., Blanco, A. \& Giancaspro, A. Map-based cloning of Qfhb.mgb-2A identifies a WAK2 gene responsible for Fusarium Head Blight resistance in wheat. Sci Reports 9, 6929 (2019).

22. Anderson, J. A. et al. DNA markers for Fusarium head blight resistance QTLs in two wheat populations. Theor. Appl. Genet. 102, 1164-1168 (2001).

23. Basnet, B. R., Glover, K. D., Ibrahim, A. M., Yen, Y. \& Chao, S. A QTL on chromosome 2DS of 'Sumai 3' increases susceptibility to Fusarium head blight in wheat. Euphytica 186, 91-101 (2012).

24. Buerstmayr, H. et al. Molecular mapping of QTLs for Fusarium head blight resistance in spring wheat. I. Resistance to fungal spread (Type II resistance). Theor. Appl. Genet. 104, 84-91 (2002).

25. Somers, D. J., Fedak, G. \& Savard, M. Molecular mapping of novel genes controlling Fusarium head blight resistance and deoxynivalenol accumulation in spring wheat. Genome 46, 555-564 (2003).

26. Zhou, W. C., Kolb, F., Bai, G. H., Domier, L. \& Yao, J. B. Effect of individual Sumai 3 chromosomes on resistance to scab spread within spikes and deoxynivalenol accumulation within kernels in wheat. Hereditas 137, 81-89 (2002).

27. Paudel, B. \& Yen, Y. Current understanding of the mechanisms of Fusarium head blight resistance and the function of Fhb1, a major FHB resistance locus in wheat: a review. Front. Genet. (in review) (2020). 
28. Bernardo, A. N., Ma, H. X., Zhang, D. D. \& Bai, G. H. Single nucleotide polymorphism in wheat chromosome region harboring Fhb1 for Fusarium head blight resistance. Mol. Breed. 29, 477-488 (2012).

29. Hao, C. et al. Association mapping and haplotype analysis of a 3.1-Mb genomic region involved in Fusarium head blight resistance on wheat chromosome 3BS. PLoS One 7, e46444 (2012).

30. Zhou, M., Hayden, M., Zhang, Z., Lu, W. \& Ma, H. X. Saturation and mapping of a major Fusarium head blight resistance QTL on chromosome 3BS of Sumai 3 wheat. J. Appl. Genet. 51, 19-25 (2010).

31. Pumphrey, M. O. Towards map-based cloning of Fusarium head blight resistance QTL Fhb1 and non-additive expression of homoeologous genes in allohexaploid wheat. PhD Dissertation, Kansas State University (2007).

32. Hofstad, A. N. et al. Examining the transcriptional response in wheat Fhb1 near-isogenic lines to Fusarium graminearum infection and deoxynivalenol treatment. The Plant Genome 9, 1 (2016).

33. Lemmens, M. et al. The ability to detoxify the mycotoxin deoxynivalenol colocalizes with a major quantitative trait locus for Fusarium head blight resistance in wheat. Mol. Plant-Microbe Interact. 18, 1318-1324 (2005).

34. He, Y. et al. Molecular characterization and expression of PFT, an FHB resistance gene at the Fhb1 QTL in wheat. Phytopathology 108, 730-736 (2018).

35. Jia, H. et al. A journey to understand wheat Fusarium head blight resistance in the Chinese wheat landrace Wangshuibai. The Crop J. 6, 48-59 (2018).

36. Su, Z. et al. A candidate gene regulates Fhb1 resistance to Fusarium head blight in wheat. In: Book of Abstracts, $5^{\text {th }}$ International Symposium on Fusarium head blight/2 ${ }^{\text {nd }}$ International workshop on wheat blast, April 6 to 9, 2016, Florianopolis, SC, Brazil, pp.23 (2016).

37. Su, Z., Jin, S., Zhang, D. \& Bai, G. Development and validation of diagnostic markers for Fhb1 region, a major QTL for Fusarium head blight resistance in wheat. Theor. Appl. Genet. 131, 2371-2380 (2018).

38. Li, G. \& Yen, Y. Jasmonate and ethylene signaling pathway may mediate Fusarium head blight resistance in wheat. Crop Sci. 48, 1888-1896 (2008).

39. Gunnaiah, R., Kushalappa, A. C., Duggavathi, R., Fox, S. \& Somers, D. J. Integrated metabolo-proteomic approach to decipher the mechanisms by which wheat QTL (Fhb1) contributes to resistance against Fusarium graminearum. PLoS One 7, e40695 (2012).

40. Gottwald, S., Samans, B., Lück, S. \& Friedt, W. Jasmonate and ethylene dependent defence gene expression and suppression of fungal virulence factors: two essential mechanisms of Fusarium head blight resistance in wheat? BMC Genomics 13, 369 (2012).

41. Xiao, J. et al. Transcriptome-based discovery of pathways and genes related to resistance against Fusarium head blight in wheat landrace Wangshuibai. BMC Genomics 14, 197 (2013).

42. Paudel, B., et alY.S.A.T.Y. Detection and quantification of Wfhb1-1 protein during FHB pathogenesis in wheat shows the role of this gene in disease resistance. In: Proceedings of 2017 National Fusarium Head Blight Forum, Milwaukee, WI, USA December 3-5, 2017, pp.50 (2017).

43. Eldakak, M. et al. A quantitative proteomics view on the function of Qfhb1, a major QTL for Fusarium head blight resistance in wheat. Pathogens 7, 58 (2018).

44. Choulet, F. et al. Structural and functional partitioning of bread wheat chromosome 3B. Science 345, 1249721 (2014).

45. Buerstmayr, H. et al. Molecular mapping of QTLs for Fusarium head blight resistance in spring wheat. II. Resistance to fungal penetration and spread. Theor. Appl. Genet. 107, 503-508 (2003).

46. Lin, F. et al. Mapping QTL associated with resistance to Fusarium head blight in the Nanda2419• Wangshuibai population. II: Type I resistance. Theor. Appl. Genet. 112, 528-535 (2006).

47. Xue, S. et al. Precise mapping Fhb5, a major QTL conditioning resistance to Fusarium infection in bread wheat (Triticum aestivum L.). Theor. Appl. Genet. 123, 1055-1063 (2011).

48. Wang, Q., Shao, B., Shaikh, F. I., Friedt, W. \& Gottwald, S. Wheat resistances to Fusarium root rot and head blight are both associated with deoxynivalenol-and jasmonate-related gene expression. Phytopathology 108, 602-616 (2018).

49. Liu, S. et al. Complex microcolinearity among wheat, rice, and barley revealed by fine mapping of the genomic region harboring a major QTL for resistance to Fusarium head blight in wheat. Funct. Integ. Genomics 6, 83-89 (2006).

50. Yu, J. B., Bai, G. H., Zhou, W. C., Dong, Y. H. \& Kolb, F. Quantitative trait loci for Fusarium head blight resistance in a recombinant inbred population of Wangshuibai/Wheaton. Phytopathology 98, 87-94 (2008).

51. Käll, L., Krogh, A. \& Sonnhammer, E. L. A combined transmembrane topology and signal peptide prediction method. J. Mol. Biol. 338, 1027-1036 (2004).

52. Schneider, C. A., Rasband, W. S. \& Eliceiri, K. W. NIH Image to ImageJ: 25 years of image analysis. Nat. Met. 9, 671 (2012).

53. Ma, M., Yan, Y., Huang, L., Chen, M. \& Zhao, H. Virus-induced gene-silencing in wheat spikes and grains and its application in functional analysis of HMW-GS-encoding genes. BMC Plant Biol. 12, 141 (2012).

54. Scofield, S. R., Huang, L., Brandt, A. S. \& Gill, B. S. Development of a virus-induced gene-silencing system for hexaploid wheat and its use in functional analysis of the Lr21-mediated leaf rust resistance pathway. Plant Physiol. 138, 2165-2173 (2005).

55. Livak, K. J. \& Schmittgen, T. D. Analysis of relative gene expression data using real-time quantitative PCR and the $2^{-\Delta \Delta C T}$ method. Methods 25, 402-408 (2001).

\section{Acknowledgements}

We thank Dr. Li Huang of Montana State University for providing the BSMV vector clones and giving us valuable suggestions regarding BSMV inoculation. This research was partially supported by funds from South Dakota Agriculture Experiment Station, and by a USDA-NIFA-AFRI award (2014-67013-21567) and a USDA-ARSUSWBSI award (FY14-HW-003) to YY.

\section{Author contributions}

Y.Y. secured the finding and directed this project; B.P., Y.Z. and Y.Y. designed these experiments; B.P., Y.Z., A.G., S.D., Y.Q., A.M., T.R. and Y.Y. conducted the experiments and collected the data; B.P., Y.Z., and Y.Y. analyzed the data and wrote the manuscript.

\section{Competing interests}

The authors declare no competing interests.

\section{Additional information}

Supplementary information is available for this paper at https://doi.org/10.1038/s41598-020-64777-9.

Correspondence and requests for materials should be addressed to Y.Y.

Reprints and permissions information is available at www.nature.com/reprints. 
Publisher's note Springer Nature remains neutral with regard to jurisdictional claims in published maps and institutional affiliations.

(c) (i) Open Access This article is licensed under a Creative Commons Attribution 4.0 International License, which permits use, sharing, adaptation, distribution and reproduction in any medium or format, as long as you give appropriate credit to the original author(s) and the source, provide a link to the Creative Commons license, and indicate if changes were made. The images or other third party material in this article are included in the article's Creative Commons license, unless indicated otherwise in a credit line to the material. If material is not included in the article's Creative Commons license and your intended use is not permitted by statutory regulation or exceeds the permitted use, you will need to obtain permission directly from the copyright holder. To view a copy of this license, visit http://creativecommons.org/licenses/by/4.0/.

(c) The Author(s) 2020 